\title{
Worsening of chronic spontaneous urticaria after intake of hot pepper
}

\author{
Murat Türk, ${ }^{1}$ Insu Yllmaz, ${ }^{1}$ Tomasz Hawro, ${ }^{2}$ Marcus Maurer ${ }^{2}$
}

\begin{abstract}
Background: Patients with chronic spontaneous urticaria (CSU) have been reported to experience increased disease activity in response to the oral intake of hot pepper (Capsicum spp.). As of now, it is unclear how common this is.

Objective: We assessed patients with CSU for the prevalence of disease worsening after the intake of hot pepper and characterized its effects on their urticaria.

Methods: A questionnaire-based survey study in adult patients with CSU and a history of hot pepper consumption was carried out at a reference center for urticaria in Turkey. CSU patients who had co-existing chronic inducible urticaria were excluded from the study.

Results: Of the eighty-five patients with CSU included in this study, 46\% (39 of 85) reported worsening of their urticaria after consuming hot pepper. Demographic features, duration of CSU and control status of urticaria were not different between patients who experienced worsening of their urticaria after the intake of hot pepper and those who did not. In affected patients, worsening of their symptoms started $1.2 \pm 1.2$ hours after the intake of hot pepper and lasted for $3.3 \pm 6.8$ hours. Symptoms disappeared significantly faster in patients who took antihistamines after worsening of their urticaria with hot pepper $(0.7 \pm 0.6$ vs. $5.8 \pm 8.8$ hours; $p=0.003)$.
\end{abstract}

Conclusions: Worsening of urticaria is common and relevant in patients with CSU in Turkey. Further studies are needed to explore if this is also the case in other geographical regions and to identify and characterize the underlying mechanisms.

Key words: Chronic spontaneous urticaria, hot pepper, capsaicin, mast cell, sensory nerve

\section{From:}

${ }^{1}$ Division of Allergy and Clinical Immunology, Department of Chest Diseases, Erciyes University School of Medicine, Kayseri, Turkey.

${ }^{2}$ Dermatological Allergology, Department of Dermatology and Allergy, Charité - Universitätsmedizin Berlin, Germany.

\section{Corresponding author:}

Marcus Maurer

Dermatological Allergology, Allergie-Centrum-Charité, Department of Dermatology and Allergy, Charité - Universitätsmedizin Berlin, Charitéplatz 1, D-10117 Berlin, Germany

E-mail: marcus.maurer@charite.de

\section{Introduction}

Chronic spontaneous urticaria (CSU) is a common and debilitating skin disorder characterized by recurrent transient and itchy wheals (hives), angioedema, or both for more than 6 weeks. ${ }^{1}$ The signs and symptoms of CSU result from the degranulation of skin mast cells and the release of histamine and other pro-inflammatory mediators. ${ }^{2}$ Most CSU patients have mast cell-activating IgG auto-antibodies and/or IgE auto-antibodies, which are held to be involved in the degranulation of their skin mast cells., ${ }^{3,4}$ Chronic infections, drugs, food intolerance and stress can lead to increased disease activity in CSU patients, ${ }^{5-9}$ although the precise mechanisms are unclear.
One possible explanation for this is that mast cell activators linked to these conditions such as complement components or neuropeptides decrease the degranulation threshold of skin mast cells. Alternatively, these signals, alone or in combination, may directly degranulate skin mast cells. Either way, further research is needed to clarify the complex pathogenesis of CSU and to identify and characterize its various aspects. ${ }^{10}$

The notion that several neuropeptides such as substance $\mathrm{P}$ are involved in the pathogenesis of CSU is supported by several independent lines of evidence. ${ }^{2}$ Stress can exacerbate CSU, and stress is linked to the release of substance $\mathrm{P}$ 
by cutaneous sensory nerves. ${ }^{5}$ In the skin, mast cells and sensory nerve endings are in close anatomical relation and interact with each other by several mediators including substance P. Substance P can degranulate skin mast cells and alter their threshold to activation. ${ }^{11,12}$ The release of histamine, tryptase, IL-31 and leukotrienes from mast cells induces the release of neuropeptides from skin nerves, which creates a positive feedback mechanism for further mast cell activation. ${ }^{13}$ Activated skin mast cells can release substance $P$, which increases this feedback even more. ${ }^{14-17}$ Patients with CSU have increased responsiveness to neuropeptides. ${ }^{11,18}$ Substance $\mathrm{P}$ has been shown to be elevated in patients with CSU, and substance $\mathrm{P}$ levels are linked to CSU disease activity. ${ }^{19}$ As of now, however, there is no direct evidence that neuropeptides contribute to the pathogenesis of CSU, and their role and relevance in the development of urticaria signs and symptoms remains unclear.

Capsaicin, the natural pungent in hot pepper (Capsicum spp.), ${ }^{20}$ acts on transient receptor potential vanilloid 1 (TRPV1) receptors on sensory nerves and induces the release of substance $\mathrm{P}$ and other neuropeptides. ${ }^{21,22}$ Activation of TRPV1 receptors on sensory nerves is responsible for the burning sensation that occurs upon skin and mucosal contact with capsaicin. ${ }^{23}$ Cases of increased disease activity in patients with CSU following oral intake of hot pepper have been reported. ${ }^{24}$ As of now, it is unclear how common this is, and the effects of hot pepper consumption in patients with CSU remain ill characterized. Therefore, in this study, we assessed CSU patients for the effects of intake of hot pepper on their disease. Specifically, we determined the prevalence of hot pepper-induced worsening of CSU. We did this for two reasons. First, we wanted to provide, for the first time, data that can help to counsel CSU patients on the risk of worsening of their CSU after hot pepper consumption. Second, oral provocation with hot pepper may serve, in future studies in selected patients, as a model for the induction of CSU symptoms and the investigation of its underlying mechanisms and the effects of treatment.

\section{Patients and Methods \\ Patients and study conduct}

This was a questionnaire-based survey study that was carried out at the urticaria reference center of Erciyes University, Allergy-Immunology Clinic, Turkey during May and August 2018. We included 85 consecutive eligible patients admitted to the outpatient clinic of our center. Inclusion criteria included 1) diagnosis of CSU, 2) hot pepper consumption history during CSU, 3) informed consent, and 4) age of 18 years or older. CSU patients who had co-existing chronic inducible urticaria (as diagnosed by a positive history of physical triggers confirmed with provocation testing) or a history of anaphylaxis after intake of hot pepper were excluded from the study. This study was approved by the institutional ethical committee (Erciyes University Medical Ethical Committee, decision no: $2018 / 541)$.

\section{Disease control and treatment history}

Disease control was measured either with the urticaria control test (UCT $\geq 12$ defined as controlled-disease) or in-clinic UAS with regard to the preceding day (range 0-6, UAS $\leq 1$ defined as controlled-disease). ${ }^{25,26}$ Treatment history of the patients was obtained from the questionnaire and/or the clinical charts.

\section{Assessment of the effects of hot pepper intake}

The effects of consuming hot pepper were assessed retrospectively, by the use of a questionnaire that consisted of 16 questions that focused on CSU disease activity, clinical manifestation and impact. Worsening of urticaria was defined as either 1) the development of novel urticarial signs and symptoms or 2) substantial worsening of existing signs and symptoms of CSU, within 3 hours after hot pepper intake. If a patient reported worsening of urticaria at least once after hot pepper intake at anytime, they were asked about 1) which urticarial signs and symptoms worsened (itch, wheals, angioedema, and burning sensation), 2) the reproducibility of this response (every time, sometimes, rarely after hot pepper intake), 3) the amount of hot pepper intake that led to worsening of urticaria (low, high), 4) the elapsed time from hot pepper intake to the beginning of the signs and symptoms, 5) antihistamine use after worsening of urticaria, 6) elapsed time for the resolution of the symptoms (with/without antihistamines), and 7) possible other similar triggers. Also, patients who reported worsening of their urticaria after hot pepper intake were asked to compare the severity of signs and symptoms before and after hot pepper intake and to quantify this change with the help of a numerical rating scale (NRS) from 1 (very minor change in the severity of signs and symptoms) to 10 (extreme change in the severity of signs and symptoms).

\section{Statistics}

Data were entered into Statistical Package for Social Sciences software version 17.0 (SPSS Inc.; Chicago, IL, USA), and analyses were made using the same software program. Kolmogorov-Smirnov test was used to compute the distribution of numerical variables; parametric variables were presented as mean \pm standard deviation and non-parametric variables were presented as median (interquartile range). When comparing continuous variables, an independent sample t test was used for parametric values and the Mann-Whitney U test for non-parametric values. Chi-square test was used to for the comparison of categorical variables. $\mathrm{p}<0.05$ was considered significant.

\section{Results \\ Patient characteristics and treatment history}

Sixty-two (73\%) of the 85 patients with CSU included in this study were female, the mean age was $39.1 \pm 11.9$ (Table 1). Four patients had co-existing atopic disease, and 6 had a comorbid autoimmune disease. The mean duration of urticaria was $3.9 \pm 4.4$ years. Seventy-three $(89 \%)$ of patients were on active treatment for their CSU at the time of the study; 31 patients (38\%) received standard dosed sgAHs, 18 patients $(22 \%)$ were on higher than standard-dosed sgAHs, 
Table 1. Patient characteristics

\begin{tabular}{|c|c|c|c|c|}
\hline & Overall & $\begin{array}{l}\text { With worsening of } \\
\text { urticaria after intake } \\
\text { of hot pepper }\end{array}$ & $\begin{array}{l}\text { Without worsening of } \\
\text { urticaria after intake } \\
\text { of hot pepper }\end{array}$ & $\mathbf{p}$ \\
\hline Patients; n (\%) & 85 & $39(46)$ & $46(54)$ & \\
\hline Age; years \pm SD & $39.1 \pm 11.9$ & $40 \pm 13.2$ & $38.4 \pm 10.7$ & 0.54 \\
\hline Female gender (\%) & $62(73)$ & $31(80)$ & $31(67)$ & 0.23 \\
\hline Co-existing diseases (\%) & & & & 0.34 \\
\hline Atopic & $4(5)$ & $3(8)$ & $1(2)$ & \\
\hline Autoimmune & $6(7)$ & $4(10)$ & $2(4)$ & \\
\hline Duration of urticaria; years (IQR) & $2(1-5)$ & $2(0.95-5)$ & $2.5(1-5)$ & 0.5 \\
\hline Treatment for CSU (\%) & $73(89)$ & $31(84)$ & $42(93)$ & 0.17 \\
\hline St-sgAH & $31(38)$ & $17(46)$ & $14(31)$ & 0.18 \\
\hline Hi-sgAH & $18(22)$ & $9(24)$ & $9(20)$ & 0.79 \\
\hline Omalizumab & $24(29)$ & $5(14)$ & $19(42)$ & 0.007 \\
\hline Disease control status at the time of study (\%) & & & & 0.81 \\
\hline Controlled & $55(67)$ & $24(65)$ & $31(69)$ & \\
\hline Uncontrolled & $27(33)$ & $13(35)$ & $14(31)$ & \\
\hline
\end{tabular}

SD: standard deviation, IQR: interquartile range, St-sgAH: standard dose second generation antihistamines, Hi-sgAH: high dose second generation antihistamines

and 24 patients $(29 \%)$ received omalizumab. At the time of study, $75 \%$ (55 of 73 ) of the patients who were treated with any of these treatment options and none of the patients without any treatment had controlled-disease (UCT $\geq 12$ and/or in-clinic UAS $\leq 1$ ).

\section{Worsening of urticaria with hot pepper is frequent in pa-} tients with CSU

Almost half of our patients (39 of 85, 46\%) reported worsening of their urticaria after consuming hot pepper. The demographic features, duration of CSU and control status of urticaria were not different between patients who reported worsening of their urticaria after the intake of hot pepper and those who did not (Table 1). The signs and symptoms of CSU that were most commonly induced or exacerbated after hot pepper intake were itchy wheals without angioedema (77\%), followed by wheals with angioedema (3.5\%), and angioedema only (2.4\%). In addition, almost half of our patients $(49 \%)$ experienced a burning sensation of the skin after hot pepper consumption. Fourteen patients with worsening of their urticaria after hot pepper intake reported similar worsening of urticaria with other spices. The mean increase in the severity of signs and symptoms after hot pepper intake was $3.9 \pm 2.2$ points on the 10 point NRS.

\section{Worsening of urticaria with hot pepper is relevant in patients with CSU}

Most patients with worsening of CSU after hot pepper intake ( 28 of $39,72 \%)$ reported that this happens every time they eat hot pepper, whereas only $10(25.6 \%)$ and one $(2.6 \%)$ patient reported that this happens only sometimes and rarely after consuming hot pepper, respectively.

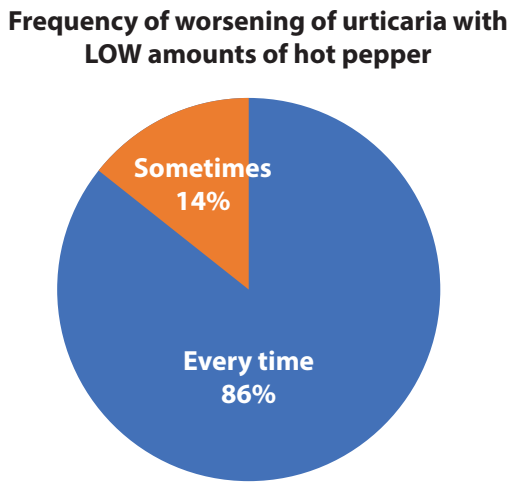

\section{Frequency of worsening of urticaria with HIGH amounts of hot pepper}

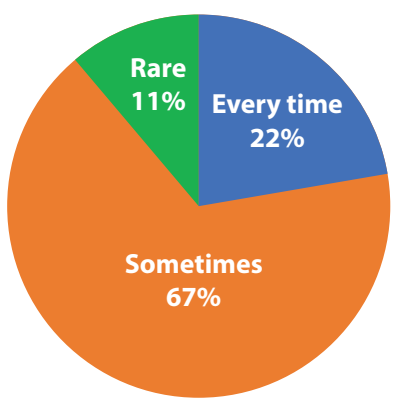

Figure 1. Pie charts demonstrating the relationship between the occurrence of disease worsening and the amount of hot pepper required. 
A

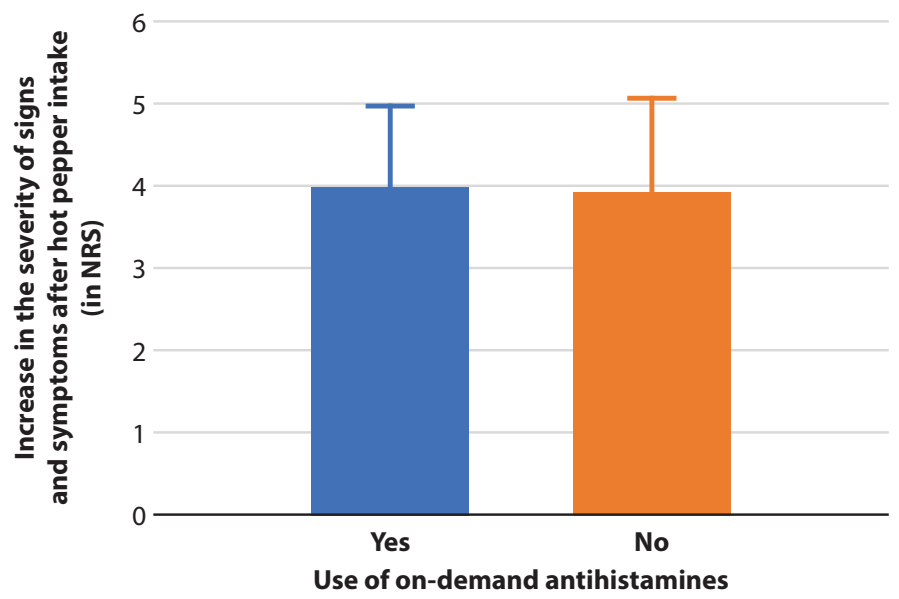

B

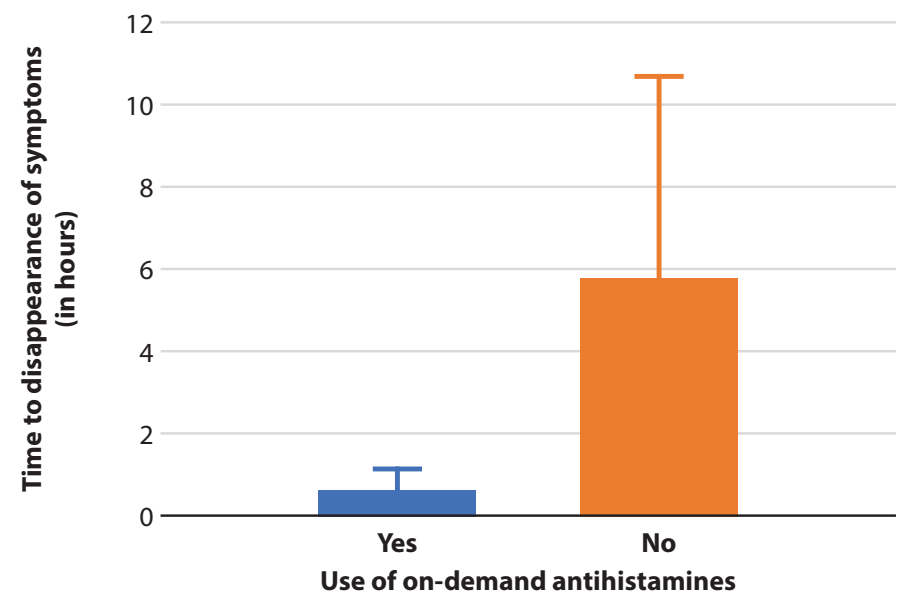

Figure 2. Mean increase in numerical rating scale (NRS) were similar in both groups (A) however, symptoms disappeared significantly faster in patients who took additional AHs as compared to those who did not (B).

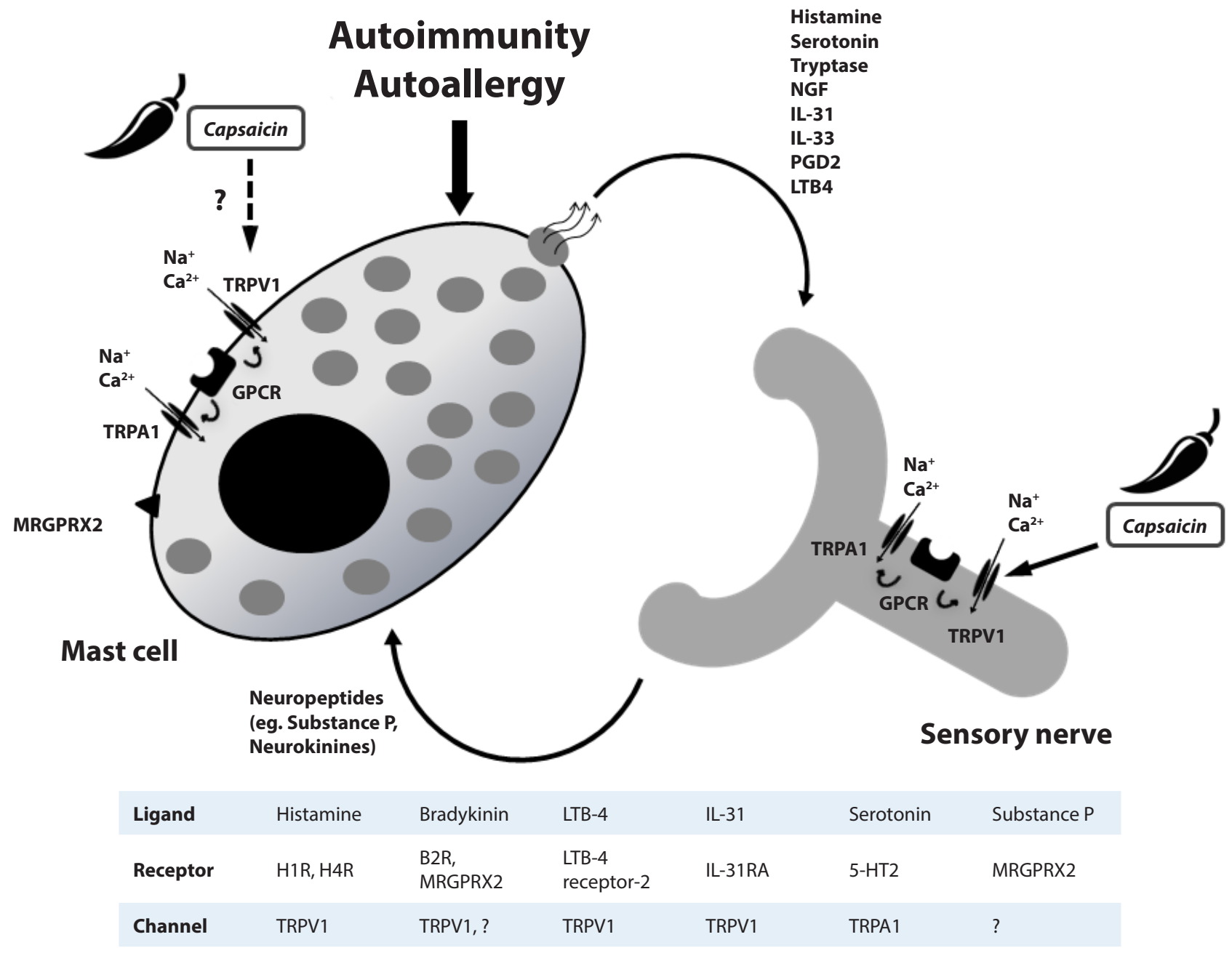

Figure 3. In the skin, mast cells and sensory nerve endings are in close anatomical relation and interact with each other by several mediators. Most of these mediators' receptors have downstream signals ending with activation of transient receptor potential channels. Capsaicin acts on TRPV1 channels on sensory nerves and induces the release of neuropeptides. Although it was shown that TRPV1 is also expressed on skin mast cells, it is still unknown whether capsaicin may directly stimulate skin mast cells. 
Most of the patients with worsening of CSU after hot pepper intake reported that low amounts of pepper were sufficient to worsen their disease. Only 9 of 39 intolerant CSU patients (23.1\%) reported worsening of the symptoms only when consuming high amounts of hot pepper. Twenty-four of the 28 CSU patients, who had worsening of symptoms every time after hot pepper intake, reported that even low amounts of hot pepper was enough for this to happen (Figure 1).

\section{Worsening of urticaria with hot pepper responds well to an- tihistamines}

Worsening of urticaria in affected patients started $1.2 \pm 1.2$ hours after the intake of hot pepper and lasted for $3.3 \pm 6.8$ hours. Twenty-one patients reported that they used additional AHs besides their regular treatments to treat the worsening of their urticaria, whereas 14 patients did not. The mean increase in the severity of signs and symptoms after hot pepper intake were similar in both groups $(4 \pm 2.1$ vs. $3.9 \pm 2.1$ NRS points, $p=0.99$ ); however, symptoms disappeared significantly faster in patients who took AHs as compared to those who $\operatorname{did}$ not $(0.7 \pm 0.6$ vs. $5.8 \pm 8.8$ hours; $p=0.003)$ (Figure 2$)$.

\section{Discussion}

In this study, we found that one of two patients with CSU who consume hot pepper, experience worsening of their urticaria after doing so. This was largely independent of the amount of hot pepper consumed and responded well to antihistamines.

Adverse reactions and intolerance to foods are frequently described by patients with CSU. Sánchez et al showed that $66 \%$ of CSU patients reported at least one adverse reaction to foods. ${ }^{24}$ They also found that $29 \%$ and $22 \%$ of the patients self reported exacerbation of CSU after eating sauces and spicy foods, respectively. In our study, 46\% (39 of 85) of the patients reported worsening of urticaria after hot pepper intake. More importantly, 24 of them reported worsening of urticaria every time they consumed hot pepper, even with low amounts.

At present, we can only speculate on the underlying mechanisms of worsening of urticaria in CSU patients after the intake of hot pepper. Previous studies revealed that food-induced urticarial reactions in patients with CSU are rarely IgE-mediated, as shown by the absence of specific IgE or negative skin prick tests. ${ }^{24,27}$ As we did not perform hot pepper skin prick tests or measurements of specific IgE, the mechanism of hot pepper-induced exacerbation of urticaria in our patients remains to be clarified. Our patients did not have a history of prior allergic reactions to hot pepper and showed occurrence of urticarial symptoms without hot pepper intake, which argues against hot pepper allergy and suggests that patients who had worsening of urticaria after hot pepper intake had a non-IgE-mediated reaction to hot pepper.

The most likely explanation, from our point of view, is that hot pepper acts as an aggravating factor that induces the degranulation of skin mast cells and/or decreases their degranulation threshold. ${ }^{7,28}$

Hot pepper contains high amounts of capsaicin, ${ }^{20}$ an irritant that produces a burning sensation upon skin and mucosal contact. ${ }^{23}$ The most frequent adverse effects of treatment with topical capsaicin formulations are application-site burning, pain, erythema and itch. ${ }^{29}$ These effects of capsaicin are held to be due to the release of neuropeptides like substance $\mathrm{P}^{23}$ from sensory nerves via the activation of TRPV1 channels, which are also expressed on mast cells. Sensory nerves of the skin, via release of substance $\mathrm{P}$ and other mechanisms, can induce the degranulation of skin mast cells and alter their activation threshold (Figure 3). ${ }^{13,30}$ Interestingly, several cases of urticarial skin reactions, i.e. wheal development, to direct and prolonged contact with hot pepper have been reported. ${ }^{31-33}$ In our study, the development of wheals in response to hot pepper consumption was experienced by 4 of 5 pepper-intolerant patients with CSU.

This study has some limitations: First, its results may be affected by its single center nature. The consumption of hot pepper and the frequency of hot pepper intolerance may be different in other geographical regions with different dietary habits. Also, patients without hot pepper intolerance may have been unwilling to consent and participate in this study, thereby introducing a selection bias. Finally, in this study, we did not perform double blind placebo-controlled oral challenge tests with hot pepper to confirm the patient-reported information obtained. We are planning to do this in future studies.

In conclusion, hot pepper-driven worsening of urticaria is common and relevant in patients with chronic spontaneous urticaria who consume hot pepper. Further studies should confirm this finding in larger and ideally global patient populations and address the underlying mechanisms. Our results should encourage physicians who treat patients with CSU to make them aware of the possibility that the intake of hot pepper can aggravate their urticaria and to advise them to abstain from its consumption if it does. Also, standardized oral provocation tests with hot pepper or capsaicin, in patients with CSU and a history of hot pepper-induced worsening of urticaria, may be a suitable model to test the efficacy of novel treatment options in preventing CSU exacerbations.

\section{Conflict of interests}

All authors declare that there are no personal or professional conflicts of interest regarding any aspects of this study.

\section{Funding}

None

\section{Acknowledgements}

Murat Türk thanks the European Academy of Allergy and Clinical Immunology for awarding him the EAACI Clinical Fellowship (2018).

\section{References}

1. Zuberbier T, Aberer W, Asero R, Latiff AA, Baker D, Ballmer-Weber $\mathrm{B}$, et al. The EAACI/GA ${ }^{2} \mathrm{LEN} / \mathrm{EDF} / \mathrm{WAO}$ guideline for the definition, classification, diagnosis and management of urticaria. Allergy. 2018; 73:1393-414.

2. Church MK, Kolkhir P, Metz M, Maurer M. The role and relevance of mast cells in urticaria. Immunol Rev. 2018;282:232-47.

3. Kolkhir P, Church MK, Weller K, Metz M, Schmetzer O, Maurer M. Autoimmune chronic spontaneous urticaria: what we know and what we do not know. J Allergy Clin Immunol. 2017;139:1772-81. 
4. Schmetzer O, Lakin E, Topal FA, Preusse P, Freier D, Church MK, et al. IL-24 is a common and specific autoantigen of IgE in patients with chronic spontaneous urticaria. J Allergy Clin Immunol. 2018;142:876-82.

5. Varghese R, Rajappa M, Chandrashekar L, Kattimani S, Archana M, Munisamy M, et al. Association among stress, hypocortisolism, systemic inflammation, and disease severity in chronic urticaria. Ann Allergy Asthma Immunol. 2016;116:344-8.

6. Zheleznov S, Urzhumtseva G, Petrova N, Sarsaniia Z, Didkovskii N, Dörr $\mathrm{T}$, et al. Gastritis can cause and trigger chronic spontaneous urticaria independent of the presence of helicobacter pylori. Int Arch Allergy Immunol. 2018;175:246-51.

7. Zuberbier T. The role of allergens and pseudoallergens in urticaria. J Investig Dermatol Symp Proc. 2001;6:132-4.

8. Jorge JS, Sánchez A, Cardona R, Sánchez J. Prevalence of drugs as triggers of exacerbations in chronic urticaria. J Investig Allergol Clin Immunol. 2019;29:112-7.

9. Cavkaytar O, Arik Yilmaz E, Buyuktiryaki B, Sekerel BE, Sackesen C, Soyer OU. Challenge-proven aspirin hypersensitivity in children with chronic spontaneous urticaria. Allergy. 2015;70:153-60.

10. Saini SS, Kaplan AP. Chronic spontaneous urticaria: the devil's itch. J Allergy Clin Immunol In Practice. 2018;6:1097-106.

11. Borici-Mazi R, Kouridakis S, Kontou-Fili K. Cutaneous responses to substance $\mathrm{P}$ and calcitonin gene-related peptide in chronic urticaria: the effect of cetirizine and dimethindene. Allergy. 1999;54:46-56.

12. Ali H. Emerging roles for mas-related g protein-coupled receptor-X2 in host defense peptide, opioid, and neuropeptide-mediated inflammatory reactions. Adv Immunol. 2017;136:123-62.

13. van der Kleij HP, Bienenstock J. Significance of conversation between mast cells and nerves. Allergy Asthma Clin Immunol. 2005;1:65-80.

14. Cevikbas F, Kempkes C, Buhl T, Mess C, Buddenkotte J, Steinhoff M. Role of Interleukin-31 and Oncostatin $\mathrm{M}$ in itch and neuroimmune communication. In: Carstens E, Akiyama T, editors. Itch: Mechanisms and Treatment. Boca Raton (FL): CRC Press/Taylor \& Francis; 2014. p. 237-252.

15. Taylor-Clark T, Nassenstein C, Undem B. Leukotriene D4 increases the excitability of capsaicin-sensitive nasal sensory nerves to electrical and chemical stimuli. Br J Pharmacol. 2008;154:1359-68.

16. Roosterman D, Goerge T, Schneider SW, Bunnett NW, Steinhoff M. Neuronal control of skin function: the skin as a neuroimmunoendocrine organ. Physiol Rev. 2006;86:1309-79.

17. Subramanian H, Gupta K, Ali H. Roles of Mas-related G protein-coupled receptor X2 on mast cell-mediated host defense, pseudoallergic drug reactions, and chronic inflammatory diseases. J Allergy Clin Immunol. 2016;138:700-10

18. Smith C, Atkinson B, Lee T, Morris R, Hayes N, Foreman J. Cutaneous responses to vasoactive intestinal polypeptide in chronic idiopathic urticaria. Lancet. 1992;339:91-3.
19. Metz M, Krull C, Hawro T, Saluja R, Groffik A, Stanger C, et al. Substance P is upregulated in the serum of patients with chronic spontaneous urticaria. J Invest Dermatol. 2014;134:2833-6.

20. Contreras-Padilla M, Yahia EM. Changes in capsaicinoids during development, maturation, and senescence of chile peppers and relation with peroxidase activity. J Agric Food Chem. 1998;46:2075-9.

21. Caterina MJ, Schumacher MA, Tominaga M, Rosen TA, Levine JD, Julius D. The capsaicin receptor: a heat-activated ion channel in the pain pathway. Nature. 1997;389:816-24.

22. Biro T, Maurer M, Modarres S, Lewin NE, Brodie C, Acs G, et al. Characterization of functional vanilloid receptors expressed by mast cells. Blood. 1998;91:1332-40.

23. Srinivasan K. Biological activities of red pepper (Capsicum annuum) and its pungent principle capsaicin: a review. Crit Rev Food Sci Nutr. 2016;56:1488-500.

24. Sánchez J, Sánchez A, Cardona R. Dietary habits in patients with chronic spontaneous urticaria: evaluation of food as trigger of symptoms exacerbation. Dermatol Res Pract. 2018;2018:237-52.

25. Hawro T, Ohanyan T, Schoepke N, Metz M, Peveling-Oberhag A, Staubach $\mathrm{P}$, et al. The urticaria activity score-validity, reliability, and responsiveness. J Allergy Clin Immunol Pract. 2018;6:1185-90.

26. Weller K, Groffik A, Church MK, Hawro T, Krause K, Metz M, et al. Development and validation of the Urticaria Control Test: a patient -reported outcome instrument for assessing urticaria control. J Allergy Clin Immunol. 2014;133:1365-72.

27. Chung BY, Cho YS, Kim HO, Park CW. Food allergy in korean patients with chronic urticaria. Ann Dermatol. 2016;28:562-8.

28. Gultekin F, Doguc DK. Allergic and immunologic reactions to food additives. Clin Rev Allergy Immunol. 2013;45:6-29.

29. Laklouk M, Baranidharan G. Profile of the capsaicin $8 \%$ patch for the management of neuropathic pain associated with postherpetic neuralgia: safety, efficacy, and patient acceptability. Patient Prefer Adherence. 2016; 10:1913-8.

30. Minnone G, De Benedetti F, Bracci-Laudiero L. NGF and its receptors in the regulation of inflammatory response. Int J Mol Sci. 2017;18:1028.

31. Feldman H, Levy PD. Hot pepper-induced urticaria while repairing a digital laceration. Am J Emerg Med. 2003;21:159.

32. Foti C, Carino M, Cassano N, Panebianco R, Vena GA, Ambrosi L. Occupational contact urticaria from paprika. Contact Dermatitis. 1997; 37:135.

33. Firoz EF, Levin JM, Hartman RD, James WD. Lip plumper contact urticaria. J Am Acad Dermatol. 2009;60:861-3. 